\title{
Snake envenomation in a north Indian hospital
}

\author{
N Sharma, S Chauhan, S Faruqi, P Bhat, S Varma
}

Emerg Med J 2005;22:1 18-120. doi: 10.1136/emj.2003.008458

\begin{abstract}
Objectives: To study the clinical profile of snake envenomation in a tertiary referral north Indian hospital.

Methods: Retrospective case note analysis of all cases of snakebite admitted to the medical emergency from January 1997 to December 2001.

Results: Of a total of 142 cases of snakebite there were 86 elapid bites presenting with neuroparalytic symptoms and 52 viper bites having haemostatic abnormalities. Some $60.6 \%$ of the cases of snakebite occurred when the patient was asleep. Urban to rural ratio was $1: 4.7$ and male to female ratio was 4.25:1. Median time to arrival at our hospital after the bite was nine hours and mean duration of hospital stay was eight days. Twenty seven cases had acute renal failure and $75 \%$ of all elapid bites required assisted ventilation. Seventeen of 119 patients who received antivenom had an adverse event. The average dose of antivenom was 51.2 vials for elapid bites and 31 vials for viper bites. Overall mortality rate was $3.5 \%$.

Conclusion: Snakebites are common in the rural population of developing countries. There is a need to educate the public about the hazards of snakebite, early hospital referral, and treatment.
\end{abstract}

$\mathrm{S}$ nakebite is a common medical emergency encountered in South Asia. In India alone 15000 people of 20000 bitten by snakes die every year. ${ }^{1}$ The principal effects of envenomation are on the nervous system, kidneys, heart, blood coagulability, vascular endothelium, and locally at the site of bite. ${ }^{1-3}$ The victims of snakebites are mainly of the rural population, who are bitten during field work and when sleeping outdoors.

The available data on epidemiology of snakebite from the Indian subcontinent are sparse because most snakebites occur in illiterate rural people who use witchcraft and traditional healers. Only cases of snakebite with severe envenomation reach the healthcare centres. This study was carried out to describe the arrival delays, clinical features, complications, and outcome of snakebites seen in a tertiary care hospital of north west India.

\section{METHODS}

This retrospective study was carried out at the Post Graduate Institute of Medical Education and Research, Chandigarh, India. This institute is a referral government hospital in north India where patients come from the states of Punjab, Haryana, Uttar Pradesh, and Himachal Pradesh. Patients included were those with:

- the presence of fang marks.

- presence of swelling, cellulitis, bleeding, blister formation at local site.

The records of snakebite victims from January 1997 to December 2001 were obtained from the records library of the hospital. This library uses the ICD-10 system for classification of diseases. The relevant details were entered on a form on which the site of bite and the type of treatment received before referral was recorded. Other details noted were the symptomatology and inhospital treatment. The resident physicians from the emergency team reported the data and cross checking of the data was carried out by two independent physicians. Neuroparalytic syndromes were defined as mild to moderate if the patient had muscle weakness and severe if ventilatory support was required. Haemostatic abnormalities were defined as bleeding from mucocutaneous sites and evident intravascular haemolysis. The time before the first dose of antivenom ws given after the snakebite, the amount of antivenom received, and adverse events were recorded. Adverse reactions to antivenom were; anaphylaxis, pyrexial reactions, and urticaria. Mortality was defined at necropsy and by death certificates. Statistical analysis of the data was performed using Instat software (Graphpad, San Diego, CA).

\section{RESULTS}

A total of 142 cases of snakebite were included in the study from the period January 1997 to December 2001. Figure 1 shows the month-wise distribution of snake bites. The number of cases of snakebite seen rose from 24 in 1997 to 39 cases in 2001. Most of the cases were young rural men with a mean age of 31.2 years (range $12-80$ years). The urban to rural ratio was $1: 4.7$ and the male to female ratio was 4.25:1. Data were complete for 136 subjects.

The data depicted in figure 1 show that most of the cases occurred during the months of May to October. Some 60.6\% of snakebites occurred at night when the patients were asleep and $23.1 \%$ during the afternoon siesta. The most frequently bitten site was the foot (38\%). Figure 2 show the sites of the bite.

Figures 3 and 4 show the clinical features. Neuroparalytic features, a hallmark of cobra and krait bites, were seen in $86(60.6 \%)$ and haemostatic abnormalities attributable to viper bites in $52(36.6 \%)$. There were no patients who had symptoms of both neuroparalysis and haemostatic dysfunction. Records of local treatment received were available in 88 patients. Seventy patients had received treatment before coming to the hospital. Among the forms of treatment received were a tourniquet tied in 16 patients, incision and drainage was performed in 14, and antivenom was given to 29. Forty patients were referred after antiseptic dressing of the bitten site. The median arrival time at the hospital after the bite was nine hours. The mean duration of hospital stay was eight days (range $=1-27$ days). At our hospital, a total of $119(83.8 \%)$ patients received equine polyvalent antivenomone vial when reconstituted gives $10 \mathrm{ml}$. Antivenom was withheld in 23 cases in the absence of systemic envenomation. The mean dose antivenom given for neuroparalytic snakebite was 51.2 vials (range $=5-190$ vials) and 32 vials (range $=1-130$ vials) for viper bites. Of the 119 patients that received antivenom, 17 patients had an adverse reaction to 


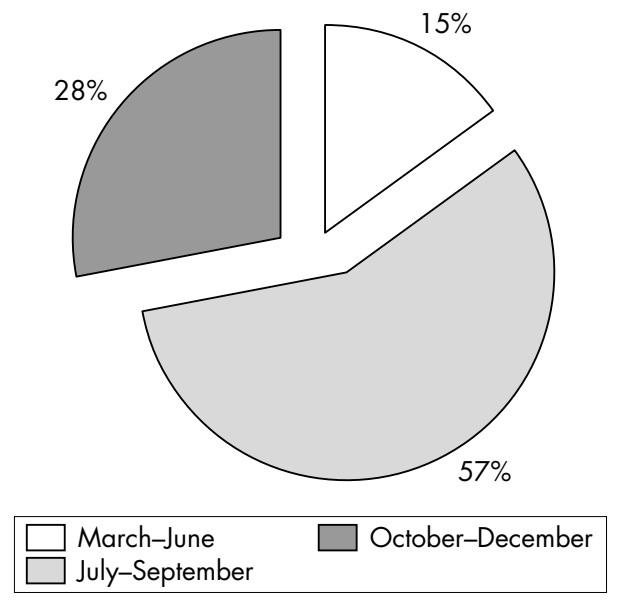

Figure 1 Pie chart of the month-wise distribution of snakebites.

antivenom (anaphylaxis (two), pyrexial reaction (6), urticaria (9)).

Of 86 patients with neuroparalytic symptoms, 65 (75.6\%) required assisted ventilation (for mean 52.8 hours). Of the 52 patients with viper bites, 39 (75\%) patients had acute renal failure and of these $33(84.6 \%)$ required dialysis. Five patients died giving a mortality rate of $3.5 \%$. Two patients died of pulmonary oedema and one each because of acute respiratory distress syndrome, cortical venous thrombosis, and disseminated intravascular coagulation.

\section{DISCUSSION}

In India, the poisonous snakes belong to the elapid family of the cobra and krait and the viper family of the Russel's viper and the saw scaled viper. Envenomation attributable to elapid bites causes paralysis of the ocular, bulbar, and limb girdle muscles. Viper bites mainly cause bleeding from mucocutaneous sites, haemolysis, acute renal failure, and occasionally shock. ${ }^{1}$

Snakebites are usually seen in people of rural areas, construction labourers, and farmers working in fields or sleeping outdoors. In this study most $(82.4 \%)$ of the snakebites occurred in people from rural areas. In concordance with the earlier reports, more snakebites occurred at night time

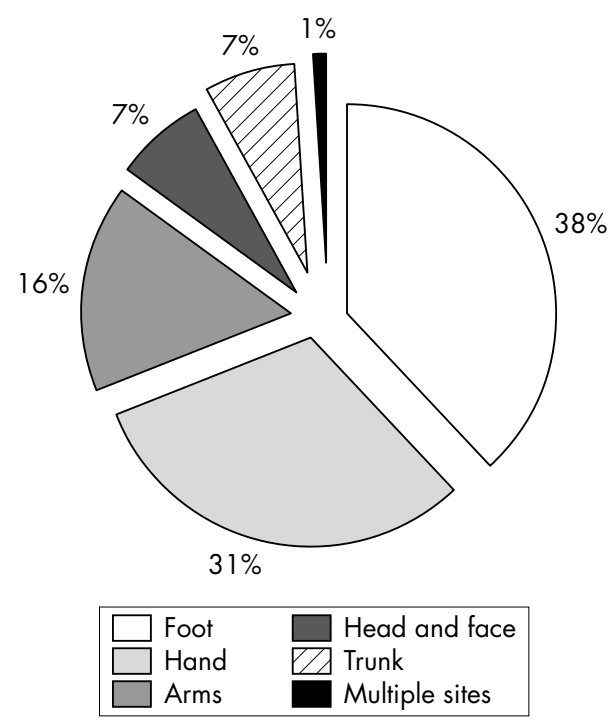

Figure 2 Pie chart of the sites of snakebite.
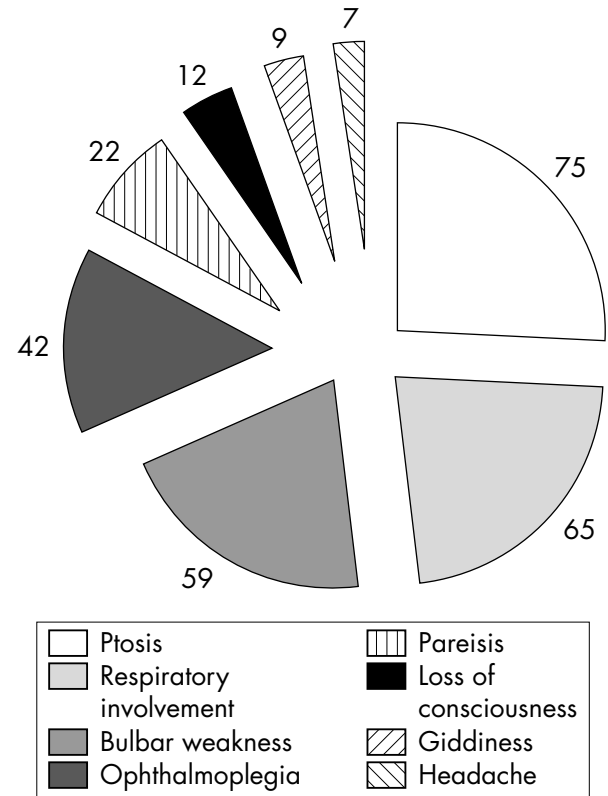

Figure 3 Pie chart showing symptoms of neuroparalytic snakebites in 86 cases.

$(60.6 \%) .^{2}$ The exposed parts of the body, mainly the hands and feet were the sites bitten in $72.5 \%$ cases. Most bites occurred during sleep (71\%) and in people engaged in manual labour in the fields $(23.2 \%)$.

Most human snakebites occur during the monsoon season because of flooding of the habitat of snakes and their prey. It is the life cycle of the natural prey of these reptiles that govern contact with humans. The breeding habits of frogs closely follow the monsoons and rats and mice are always in close proximity to human dwellings. In our study there was a higher incidence of snakebites during the monsoon season. From another south Indian study snakebites abound during the months of October to December (33\%) and May to July $(67 \%) .^{3}$
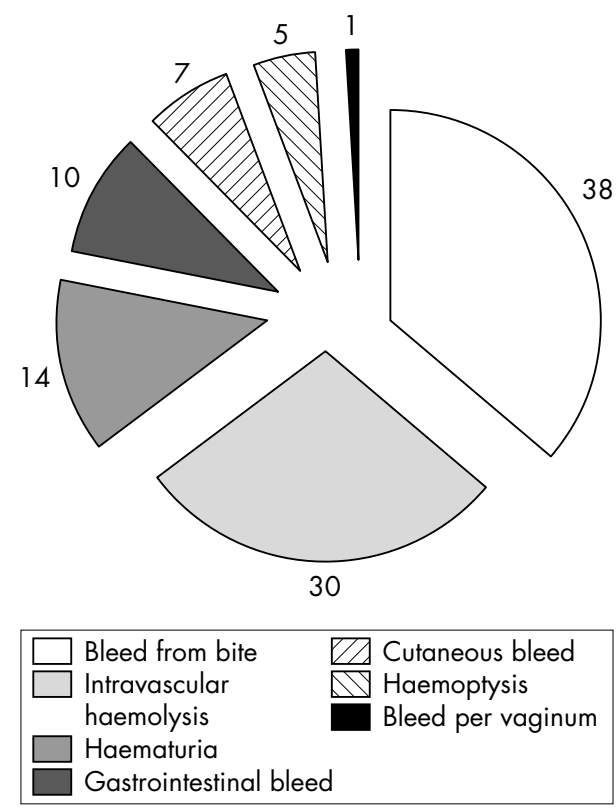

Figure 4 Pie chart showing symptoms of viper bites in 52 cases. 
Treatment for snakebite is care of the bitten site and antivenom. Seventy two of our patients had received local treatment of the bitten site-that is, tourniquet, incision and drainage, and indigenous herbal medicines. These practices are not currently recommended in the treatment of snake envenomations. ${ }^{4}$ The median arrival time after the snakebite was nine hours. A prolonged arrival delay was seen in patients presenting with acute renal failure to this hospital. This can be explained on a geographical basis as all patients who developed acute renal failure came from the state of Himachal Pradesh, where access to villages by common modes of transport is still rudimentary. This delay in arrival can also be attributed to a lack of awareness of the hazards of snakebite, an unrelenting belief in the traditional system of medicines, and a lack of proper primary healthcare facilities.

Most snakebites in our study were neuroparalytic (60.6\%) followed by bites causing haemostatic dysfunction (36.6\%). The neuroparalytic symptoms seen were; in descending order ptosis, respiratory muscle involvement, oculobulbar weakness, and limb weakness (fig 1). The reported incidence of neurological symptoms from two Sri Lankan studies on neuroparalytic snakebites was ptosis in $70 \%-85 \%$, respiratory muscle weakness in 18\%-45\%, ophthalmoplegia in 53\%-75\%, and limb weakness in $27 \%-54 \%$ respectively. ${ }^{5}$

Of 52 patients presenting with haemostatic abnormalities, bleeding from the vipers bites was most frequent followed by intravascular haemolysis and haematuria. In other studies, haemorrhagic syndromes after snakebites have occurred in $47.8 \%-90 \% .^{78}$ This variation in haemorrhagic manifestations can be explained by the subspecies differences of snake venoms.

Antivenom was given to 119 patients only. Although the dose of antivenom is not yet fixed, the indications are well known. ${ }^{8}$ The mean dose of antivenom used was 51.2 vials for elapid bites and 31 vials for viper bites. Adverse reactions to antivenom were seen in $14.2 \%$, but there were no deaths. The death rate in our study was $3.5 \%$ and compares well with the death rate from other studies across India. ${ }^{2} 37$

In conclusion, snakebite mainly afflicts the rural men of developing countries. Availability of antivenom at primary healthcare centres and rapid transportation facilities may change the morbidity associated with snakebites. There is an urgent need to educate the rural population about the hazards and treatment of snakebites. Also, randomised controlled trials are needed to investigate the issue of rationale of antivenom treatment.

\section{Authors' affiliations}

N Sharma, S Chauhan, S Faruqi, P Bhat, S Varma, Postgraduate Institute of Medical Education and Research, Chandigarh, India

Correspondence to: Dr N Sharma, Medicine, Postgraduate Institute of Medical Education and Research, Chandigarh 160012, India; nsharma@glide.net.in

Accepted for publication 12 September 2003

\section{REFERENCES}

1 Reid HA, Theakston ROG. The management of snake bite. Bull World Health Organ 1986;61:885-95.

2 Virmani SK, Dutt OP. A profile of snake bite poisoning in Jammu region. J Indian Med Assoc 1987;85:132-4.

3 Kulkarni ML, Anees S. Snake venom poisoning-experience with 633 cases. Indian Pediatr 1994;31:1239-44.

4 Warrel DA. First-aid treatment of snake bite-Harveian Oration. Lancet 2001;358:1986.

5 Kularatne SAM. Common krait (Bungarus coerulus) bite in Anuradhapura, Sri Lanka: a prospective clinical study 1996-98. Postgrad Med J 2002; 78:276-80

6 Seneviratne U, Dissanayake S. Neurological manifestation of snake bite in Sri Lanka. J Postgrad Med 2002;48:275-8.

7 Saini RK, Sharma S, Singh S, et al. Snake bite poisoning. A preliminary report. J Assoc Physicians India 1984;32:195-7.

8 Warrel DA. The clinical management of snake bites in the South East Asian region. Southeast Asian J Trop Med Public Health 1999;30(suppl):S1-67. 